\title{
Les foraminifères bio-indicateurs d'impact dans les environnements estuariens et portuaires
}

\author{
Debenay J-P. ${ }^{1}$, Geslin E. ${ }^{1}$, Guillou J-J. ${ }^{2}$ \& Casamajor M.N. de ${ }^{3}$ \\ 1) Laboratoire de Géologie, EA2644, 2 Bd Lavoisier, 49045 Angers \\ cedex - et LÉBIM, 85350 île d'Yeu. \\ 2) Laboratoire de Géologie et EA2644, Fac. Sciences, 2 rue de la \\ Houssinière, 44072 Nantes cedex 3. \\ 3) Laboratoire Ifremer, Station d'Hydrobiologie, B.-P.3, 64310 \\ Saint-Pée-sur-Nivelle
}

Résumé

Ce travail donne les grands traits de la répartition des foraminifères dans les systèmes paraliques et décrit, sur deux exemples, les impacts anthropogéniques. Malgré la diversité de ces systèmes, les foraminifères se répartissent naturellement selon quelques gradients majeurs. Des anomalies de cette répartition et/ou des malformations des tests indiquent des stress naturels ou anthropogéniques. Ainsi, une augmentation anormale de la densité des peuplements, de la richesse spécifique et de la taille des individus indique une pollution organique; une pollution chimique produisant les effets inverses. Cribroelphidium excavatum et Haynesina germanica caractérisent les zones polluées chimiquement alors que les bolivinidés ont leur abondance relative maximum dans les sédiments fins des ports de plaisance.

\begin{abstract}
This work is based on about 2,000 samples. It provides a synthetic description of the distribution patterns of foraminiferal assemblages in paralic environments and shows on two examples the effect of anthropogenic impacts. It shows that despite the great diversity of ecological parameters, foraminiferal assemblages are naturally distributed along a few major gradients. Anomalies in these distribution patterns or morphological abnormalities of the tests indicate anthropogenic or natural stresses. An increase in the number of individuals, specific richness and test size indicate organic pollution while chemical pollution as an inverse effect. In Port-Joinville harbour, Cribroelphidium excavatum and Haynesina germanica characterize the areas submited to the strongest chemical impact while the bolivinids have their maximum relative abundance in the fine sediments of the marina.
\end{abstract}

\section{Introduction}

Le suivi de l'impact des aménagements estuariens ou portuaires sur l'hydrosystème est rendu difficile par la variabilité considérable de ces environnements au cours des cycles saisonniers et de marées, surtout en domaine macrotidal. Il est difficile et coûteux d'étudier ces impacts sur la seule base 
d'analyses chimiques des eaux, car cela exigerait un suivi quasi continu en des points multiples. Les bio-indicateurs benthiques sont beaucoup mieux adaptés car ils donnent une image moyenne (intégrative) des caractéristiques écologiques de leur milieu de vie. Dans les eaux douces, plusieurs organismes (diatomées, larves de chironomes, etc.) sont utilisés pour le calcul d'indices biotiques. Aucun indice de ce type n'existe dans les environnements paraliques bien qu'ils soient soumis à de multiples pollutions. Les foraminifères benthiques qui s'y développent ont pourtant un potentiel unique :

1) Certaines espèces typiques des environnements paraliques ont une répartition quasi universelle, ce qui autorise des études comparatives globales.

2) Ils vivent généralement sur ou dans le sédiment qui reçoit et adsorbe les polluants et ont des possibilités de déplacement très limitées. Ils sont donc directement affectés par les pollutions qui existent en un lieu donné.

3) Ils ont un cycle de reproduction de quelques semaines à quelques mois selon les espèces et réagissent donc rapidement aux changements de l'environnement.

4) Leurs tests peuvent subsister après la mort des cellules. Les thanatocénoses donnent une image moyenne des conditions écologiques en effaçant les variations de courtes périodes comme les cycles de marée, propriété particulièrement intéressante dans les ports et les estuaires où le milieu est d'une variabilité extrême.

5) Ils sont présents en grande quantité dans la plupart des sédiments superficiels (jusqu'à 50000 tests pour $50 \mathrm{~cm}^{3}$ ), fournissant ainsi une base statistique frable, même pour un petit volume de sédiment.

L'étude des foraminifères peut être faite à différentes échelles d'organisation, ce qui permet d'accumuler les informations sur les caractéristiques environnementales. À l'échelle de l'individu, les anomalies morphologiques du test résultent soit de stress naturels, soit de stress induits par la pollution, notamment par les métaux lourds. À l'échelle de l'espèce, le comportement peut être différent avec, soit des exigences écologiques strictes, soit une tolérance aux stress, en particulier anthropogéniques. À l'échelle du peuplement, des perturbations dans l'organisation naturelle des peuplements permettent de mettre en évidence l'effet des stress naturels ou anthropogéniques. Finalement, ces études peuvent être utilisées, d'une part, pour caractériser l'environnement dans son organisation naturelle et, d'autre part, pour mettre en évidence des perturbations de l'écosystème. De plus, la taille des foraminifères, généralement comprise entre 0,05 et $0,5 \mathrm{~mm}$ permet une observation au stéréomicroscope, sans préparation spéciale.Leur étude est donc plus simple, plus rapide et moins coûteuse que celle d'organismes plus petits (diatomées par exemple).

Au cours des dernières années, de nombreux travaux ont été menés pour développer l'utilisation des foraminifères comme biomarqueurs ou bio-indicateurs de pollution côtière. Ils s'intéressent généralement aux zones exposées à des pollutions industrielles, agricoles ou domestiques conduisant à des contaminations par les hydrocarbures, les métaux lourds, les pesticides ou un excès de matière organique. Leur objectif principal est de définir les effets de la pollution sur la répartition des foraminifères et sur les variations morphologiques (par exemple, Bandy et al., 1964 ; Alve, 1995 ; Sharifi et al., 1991 ; revue in Yanko et al., 1999). Certaines de ces études s'intéressent plus particulièrement aux zones portuaires 
(Murray, 1968 ; Rouvillois, 1972 ; Naidu et al., 1985 ; Debenay et al., 1997).

L'objectif de cet article est de présenter la répartition générale des peuplements de foraminifères dans les milieux estuariens et portuaires et de montrer par deux exemples concrets comment ces peuplements peuvent être perturbés par des impacts anthropogéniques.

\section{Méthodes}

Sur l'estran, les sédiments sont prélevés à marée basse, à la main. Dans les zones subtidales, les prélèvements sont effectués avec une benne à prélèvements, depuis une petite embarcation. Pour toutes les études de peuplements actuels, seule la couche superficielle du sédiment $(0,5$ à $1 \mathrm{~cm})$ est récoltée. L'échantillon est immédiatement fixé à l'alcool. La taille des échantillons est toujours suffisante pour permettre de disposer de $50 \mathrm{~cm}^{3}$ de sédiment au laboratoire.

Les sédiments sont traités au laboratoire selon les techniques habituelles : lavage sur tamis de 350 et $50 \mu \mathrm{m}$; coloration au Rose Bengale pour l'étude des biocénoses ; séchage à $50{ }^{\circ} \mathrm{C}$; séparation des foraminifères par flottage sur tétrachlorure de carbone; observation sous la loupe binoculaire et/ou au microscope électronique à balayage ; détermination des espèces et comptage des peuplements ; analyses statistiques si nécessaire ; identification des espèces clés.

\section{Organisation naturelle des peuplements de foraminifères dans les estuaires}

De nombreuses études ont été faites sur les foraminifères des environnements paraliques. Certaines décrivent les répartitions verticale et/ou horizontale des peuplements (Scott \& Medioli 1986 ; Debenay 1990 ; Hayward \& Hollis 1994). D'autres proposent une comparaison entre les assemblages de différentes regions (revue in Murray, 1991).

Les microfaunes des environnements paraliques sont soumises à la dualité des influences marines et continentales dont l'intensité respective change à un rythme variable. Dans la zone de transition des estuaires macrotidaux, le passage rapide d'eaux à caractère marin à des eaux à caractère continental provoque sur les organismes un stress considérable. Sur l'estran, les variations brutales de température, salinité, pH, oxygénation, etc. qui se produisent au cours de l'émersion provoquent également un très fort stress pour les microfaunes endopéliques, épipéliques et épiphytes.

L'étude de près de 2000 échantillons dans différents estuaires, ports et lagunes a permis de mettre en évidence les tendances générales d'évolution des peuplements de foraminifères. Dans les estuaires, cette évolution se fait suivant la transition eaux marines-eaux continentales et dans les marais maritimes adjacents, suivant la transition milieu aquatique-milieu terrestre (fig. 1). 


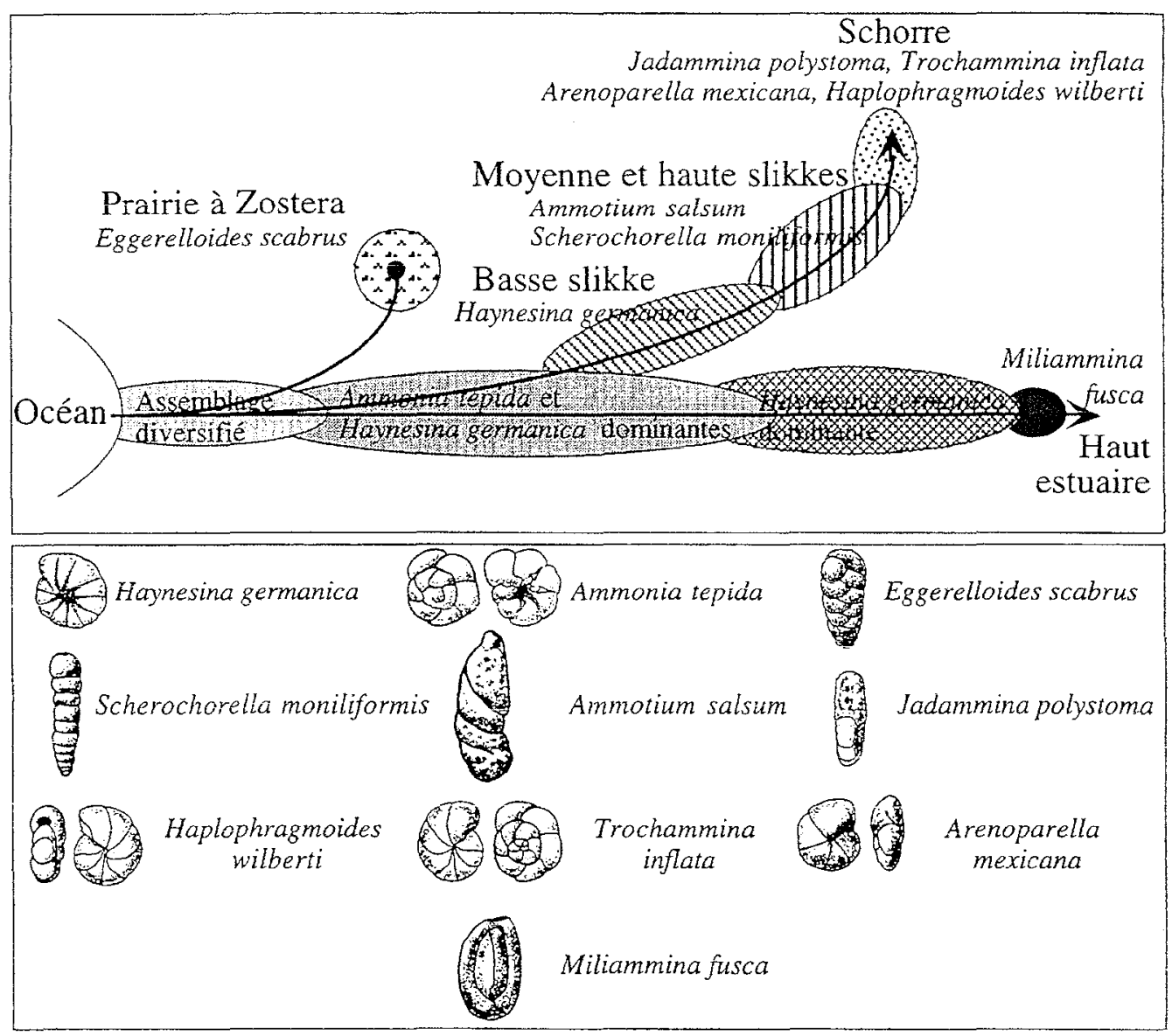

Figure 1 : Organisation naturelle des peuplements de foraminifères dans un estuaire Figure 1: Natural distribution of foraminiferal assemblages in an estuary

La transition eaux marines-eaux continentales se traduit par une diminution rapide de la richesse spécifique de l'aval vers l'amont. De nombreuses espèces disparaissent alors et Ammonia tepida et Haynesina germanica, puis H. germanica seule, deviennent dominantes. Lorque l'influence continentale est trop forte, ces espèces calcaires disparaissent et sont remplacées par Miliammina fusca. Cette forme agglutinée est la dernière espèce de foraminifères avant la disparition totale de ceux-ci vers l'amont. En bordure de marais, $H$. germanica, présente sur la basse slikke, est l'espèce à test calcaire qui supporte le mieux l'émersion. Elle est remplacée sur la haute slikke par des espèces à test agglutiné : Ammotium salsum et Scherochorella moniliformis. Sur le schorre, les espèces agglutinées dominent avec Jadammina polystoma, Trochammina inflata, Arenoparella mexicana et Haplophragmoides wilberti (Redois \& Debenay, 1996). Une espèce carbonatée (Cribroelphidium williamsoni) peut également se développer ponctuellement dans les mares du schorre. 


\section{Perturbations de l'organisation naturelle par des activités anthropiques}

\subsection{Exemple de l'estuaire de l'Adour (France)}

Les perturbations des microfaunes de foraminifères dues aux activités humaines ont été recherchées dans l'estuaire de l'Adour (Casamajor \& Debenay, 1995). Le débit moyen annuel de l'Adour est de $326 \mathrm{~m}^{3} \cdot \mathrm{s}^{-1}$. L'action de la marée se fait sentir jusqu'à environ $30 \mathrm{~km}$ en amont mais le débit fluvial limite fortement l'intrusion saline pendant les périodes de crue (printemps et automne). La salinité de la zone étudiée varie de 5 à $18 \%$, avec la présence d'un coin salé (Elbée, 1982). L'estuaire de l'Adour présente de très fortes concentrations en cadmium et des concentrations supérieures à la moyenne nationale en mercure et en PCB (Claisse, 1991).

Plusieurs sites localisés dans la zone intertidale ont été sélectionnés, soit à proximité des sources de pollution, soit sur des zones neutres où les pollutions sont plus largement diluées (fig. 2).

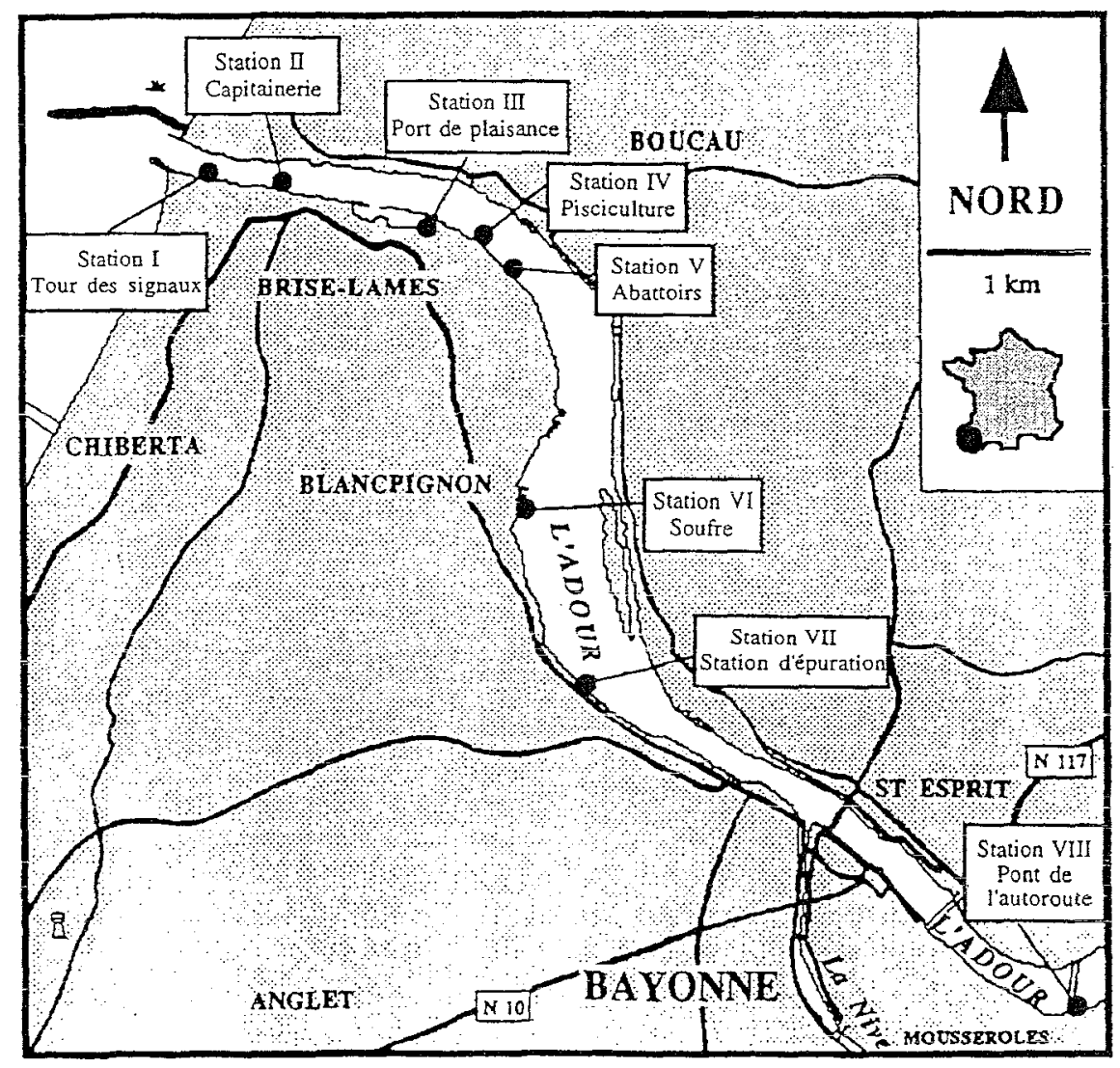

Figure 2: Situation des échantillons dans l'estuaire de l'Adour

Figure 2: Location of the samples collected in the estuary of Adour River

De l'aval vers l'amont, des prélèvements ont été réalisés en huit stations : Station I, à proximité immédiate de l'embouchure ; Station II, point intermédiaire où 
l'influence marine est élevée; Station III, zone de carénage recouverte d'une épaisse couche de sédiment à l'intérieur du port de plaisance; Station IV, rejet continu et sans traitement préalable des eaux de la pisciculture issues de pompages. Plus salées, moins turbides et plus chaudes que les eaux de l'Adour, ces eaux sont chargées du surplus de granulés utilisés pour l'alimentation des poissons, de déjections et des bactéries associées (pH et taux d'oxygène dissous bas) ; Station V, rejets des abattoirs composés de sang et d'autres matières organiques d'origine animale; Station VI, proximité du stockage du soufre de Lacq, apports de soufre par le vent et l'eau de ruissellement; Station VII, rejet des eaux de la station d'épuration du district Bayonne-Anglet-Biarritz qui présentent les mêmes tendances, moins marquées, que celles de la station IV; Station VIII, la plus amont sans source particulière de pollution. Les six premières stations ont été prélevées à 6 reprises au mois d'Août 93 et du mois de Novembre 93 au mois de Mars 94 . Les deux dernières stations les plus amont ont été échantillonnées au cours des quatre dernières séries de prélèvement.

Les tendances généraíes observées correspondent à la répartition naturelle des microfaunes en estuaire avec une diminution vers l'amont dü nombre d'individus et de la richesse spécifique, et la présence de Miliammina fusca dans les stations les plus en amont. Cette tendance est perturbée dans les stations soumises à des rejets anthropogéniques, sauf la station VI où aucun impact $n^{*}$ a été détecté (fig. 3). Seules les stations perturbées seront prises en compte ici.

La station III (port de plaisance) est caractérisée par une faible diversité spécifique et une très faible densité du peuplement, dominé par Miliammina fusca. Cette espèce étant toujours associée à une très forte influence des eaux douces, il est possible que les eaux utilisées pour le carénage des bateaux aient un rôle important. Toutefois, Miliamminafusca a aussi été signalée sur divers sites pollués tels qu'un bassin de stockage d'eaux usées en Caroline du Nord (Le Furgey \& St Jean, 1976) et un estuaire suédois pollué par les eaux usées et les métaux lourds où elle est une des premières espèces à recoloniser le milieu après récupération (Murray, 1991). Sa dominance dans cette station peut donc aussi être due aux pollutions chimiques (hydrocarbures et peintures anti-recouvrement). Cette deuxième hypothèse semble être étayée par la présence de tests anormaux. En effet, plusieurs auteurs ont noté que la présence de contaminants peut affecter la morphologie des foraminifères (Revue in Alve, 1991 et Yanko et al., 1999). Toutefois, les apports en matière organique peuvent également être à l'origine d'anomalies morphologiques des tests (revue in Geslin, 1999) ainsi que des stress dus à des causes naturelles. L'hypersalinité ou les changements de salinité peuvent avoir un rôle important dans la formation d'anomalies morphologiques (Stouff et al., 1999). Dans le cas de l'Adour, les tests anormaux n'ont pas été étudiés systématiquement, mais leur abondance a été remarquée dans la station III où deux stress sont possibles: l'eau douce du carénage et les pollutions chimiques résultant de cette activité. 


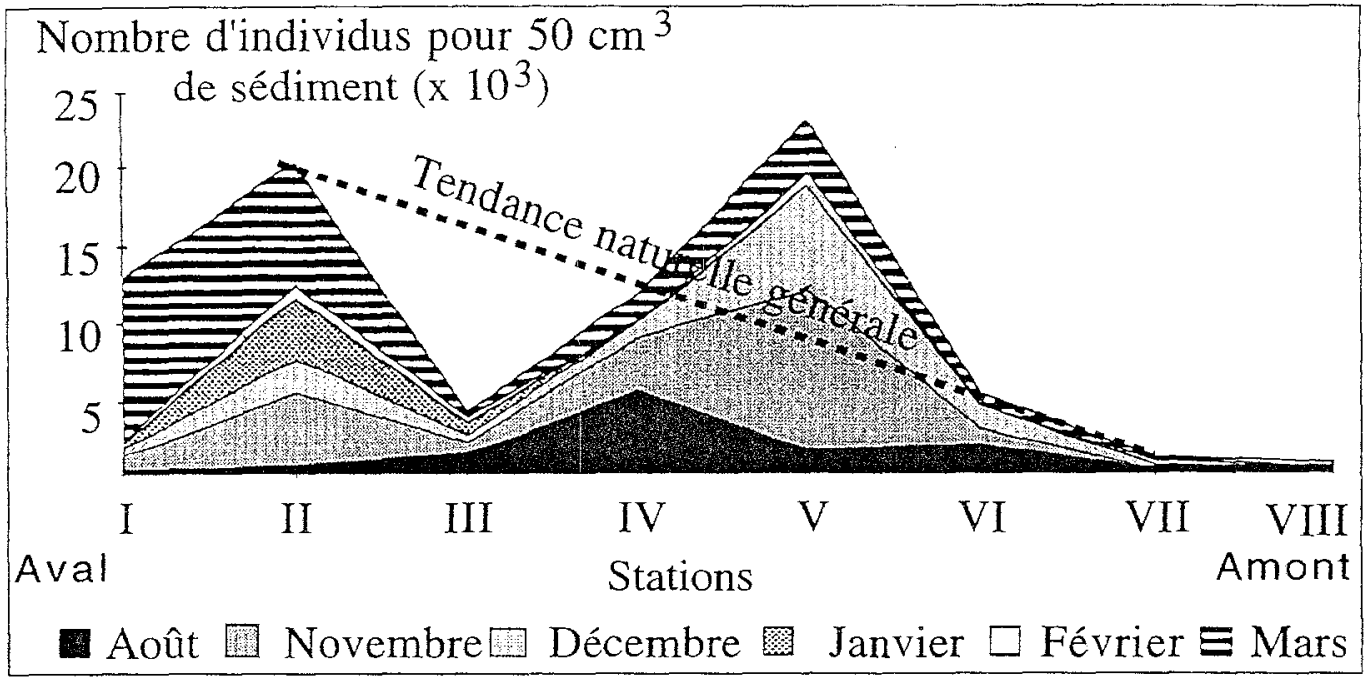

Figure 3: Densité des peuplements de foraminifères dans l'estuaire de l'Adour Figure $3:$ Number of specimens in the foraminiferal assemblages of Adour River

Les peuplements de la station IV (rejet de la pisciculture) sont caractérisés par des individus de taille supérieure à la moyenne, ceux de la station V (rejets des abattoirs) par la plus forte densité d'individus. Ces observations sont en accord avec des données de la littérature qui constatent qu'un apport supplémentaire en nutriments par des effluents organiques peut provoquer une augmentation de la densité du peuplement avec présence de tests anormalement grands. En plus de l'apport organique proprement dit, la prolifération bactérienne qui en résulte peut être favorable au développement des foraminifères qui consomment une quantité plus ou moins importante de bactéries (Muller \& Lee, 1969). Toutefois, Le Furgey \& St Jean (1976), constatent qu'au delà d'une certaine concentration en bactéries $\left(10^{5} \cdot \mathrm{ml}^{-1}\right)$, les fonctions vitales des foraminifères peuvent être inhibées. De plus, une intense dégradation de la matière organique par les bactéries conduit à des conditions anoxiques et à une forte mortalité des microfaunes. Ceci peut expliquer la présence fréquente d'une zone azoïque ou d'une zone très appauvrie à proximité immédiate des sources de pollution, aussi bien dans les zones côtières que dans les estuaires (Bandy et al., 1964 ; Schafer, 1970 ; LeFurgey \& StJean, 1976 ; Naidu et al., 1985 ; Schafer et al., 1991). Ce stade défavorable n'est pas atteint dans l'estuaire de l'Adour, probablement en raison des forts courants qui dispersent en grande partie les effluents.

Le peuplement de la station VII (rejets de la station d'épuration) ne montre pas d'enrichissement particulier, ni de croissance anormale des tests. Ceci est probablement dû au fait que cette station se trouve à la limite extrême de survie des foraminifères vers l'amont, dans des eaux fortement dessalées.

\subsection{Exemple d'un port : le port de Port-Joinville (France)}

L'exemple choisi est celui du port de Port-Joinville (île d'Yeu). Ce port situé sur une île reçoit un apport très limité d'eau douce. Il subit (1) une pollution organique 
due aux activités de pêche et au séjour de bateaux de plaisance (environ 450 pendant les mois d'été) et (2) une pollution chimique due au carénage des bateaux, aux rejets d'hydrocarbures et aux effets des peintures anti-recouvrement. La pollution est donc maximale en été, à proximité des zones de carénage. Dix-sept stations ont été échantilonnées. Les analyses de TBT and TPhT ont été réalisées par les laboratoires de l'Ifremer; les analyses de TOC et de métaux lourds (Al, As, Cd, $\mathrm{Cr}, \mathrm{Cu}, \mathrm{Hg}, \mathrm{Ni}, \mathrm{Pb}, \mathrm{Zn}$ ) et de $\mathrm{PAH}$ et $\mathrm{PCB}$ ont été faites à l'Institut Pasteur de Lille. Des analyses de corrélation entre les principales espèces de foraminifères et les polluants ont été réalisées, de même que des analyses statistiques sur les peuplements.

Cent-dix-huit espèces de foraminifères ont été récoltées. Les principales caractéristiques du peuplement sont : l'abondance relative maximum des bolivinidés dans les sédiments fins du port de plaisance; l'abondance relative maximum de Cribroelphidium excavatum et Haynesina germanica dans les zones les plus internes du port de plaisance et dans le bassin à flot; la présence de plus de $10 \%$ d'Elphidium pulvereum dans le bassin à flot; la présence de plus de $20 \%$ des deux espèces épibiotes Planorbulina mediterranensis et Lobatula lobatula dans la partie centrale du port et de plus de $20 \%$ de Textularia truncata dans la zone externe du port.

Les bolivinidés sont connus pour tolérer un appauvrissement du milieu en oxygène (Murray, 1991), leur morphologie allongée et aplatie étant considérée comme une adaptation à ce type d'environnement (Bernhard, 1986). En domaine océanique, ils sont généralement dominants dans la zone d'oxygène minimum ou dans les zones d'upwelling (Mullins et al., 1985). Toutefois, la teneur relativement élevée en oxygène des eaux de fond et la couleur brun clair des sédiments n'indiquent pas un appauvrissement en oxygène dans le port de plaisance. La forte abondance de ce groupe a déjà été rapportée dans des conditions similaires dans le port de plaisance de La Turballe (Debenay et al., 1997). Une étude d'impact faite dans le port de Pornichet a montré que l'effet des effluents issus des bateaux de plaisance était "à peu près négligeable" (Legrand \& Robbe, 1994). Il est toutefois possible d'émettre l'hypothèse d'un effet spécifique de ces effluents sur les foraminifères, favorisant le développement des bolivinidés par un enrichissement en phosphate, par exemple. Des analyses complémentaires devront être réalisées pour vérifier cette hypothèse. Dans le groupe des bolivinidés, les analyses de corrélations ont montré que Bolivina pseudodoplicata et Bolivina sp. 1 sont corrélées positivement avec Al et Brizalina variabilis avec $\mathrm{Cd}$.

Cribroelphidium excavatum est souvent considéré comme une espèce tolérante à la plupart des contaminants, très adaptable et opportuniste, capable d'adopter un mode de vie endopélique ou épipélique selon les circonstances. Il a été observé en association avec des espèces agglutinées également très résistantes ou avec Haynesina germanica (Sharifi et al., 1991 ; Alve 1995). Ces auteurs ont établi que $C$. excavatum est l'espèce la plus tolérante à la pollution par les métaux lourds, suivie de $H$. germanica et $A$. beccarii dans cet ordre. $C$. excavatum et $H$. germanica ont également été signalées comme les espèces pionnières dans la recolonisation de l'estuaire de la Gota après récupération (Cato et al., 1980). L'augmentation notable de l'abondance relative de ces deux espèces dans les zones les plus internes du 
port, à proximité des zones de carénage, est donc probablement l'indice d'un fort impact anthropique dans ces zones (fig. 4).

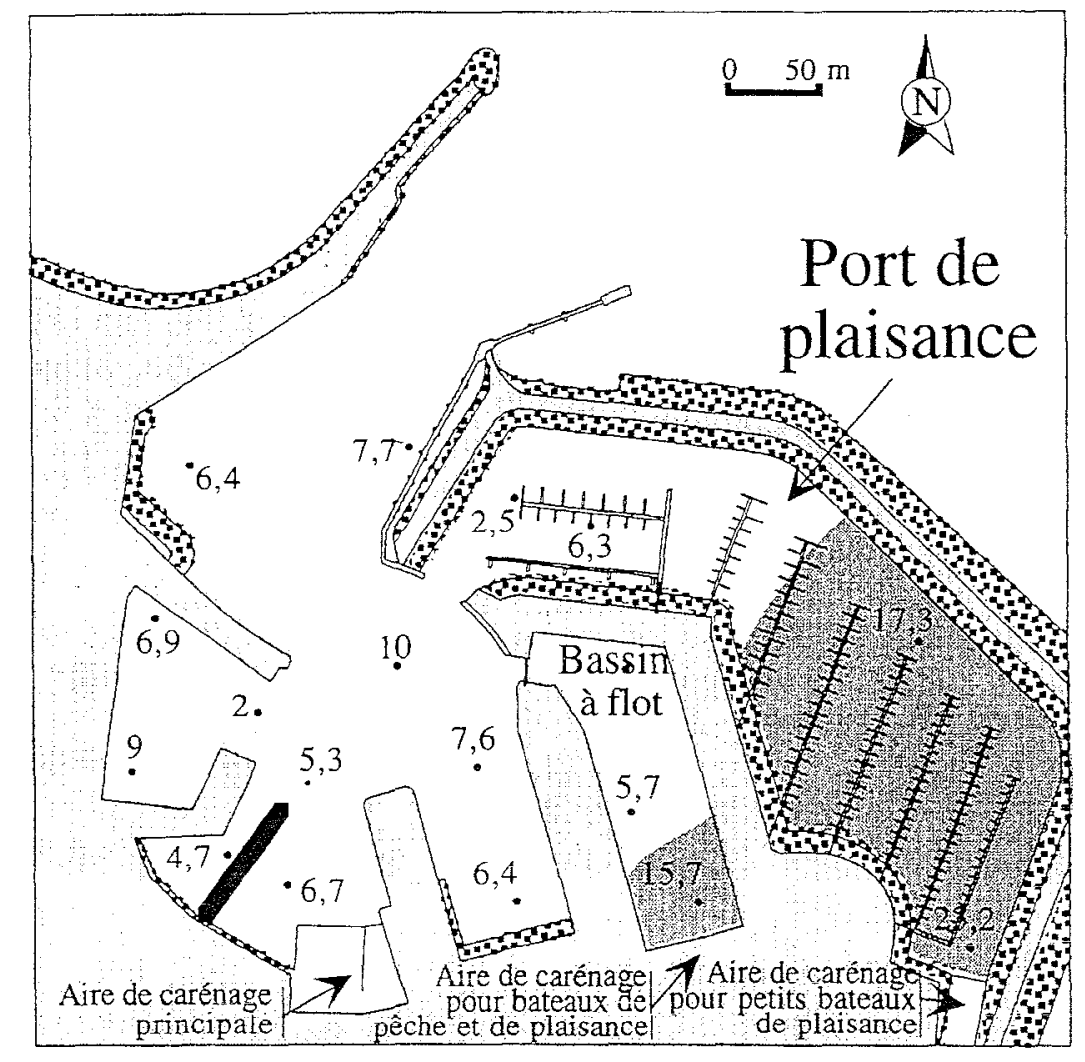

Figure 4: Abondance relative de C. excavatum dans le port de Port-Joinville Figure $4:$ Relative abundance of $\mathrm{C}$. excavatum in Port-Joinville harbour

De plus, C. excavatum est fortement corrélé positivement avec l'arsenic et $H$. gemanica avec le plomb. La faible abondance relative de ces espèces en face de l'aire de carénage principale, la plus fortement contaminée en cuivre, plomb, zinc et TBT est probablement due au fait que cette zone est soumise à une forte agitation par les hélices des navettes qui assurent la liaison avec le continent. Il est probable que cette agitation, en provoquant un brassage périodique du sédiment superficiel, agit sur la bio-disponibilité des contaminants.

L'espèce Elphidium pulvereum présente un cas à part. C'est une espèce épiphyte dont la présence dans le bassin à flot résulte du développement d'algues vertes qui lui servent de support. Ces algues se développent en raison de la faible variation du niveau des eaux au cours du cycle de marée. La présence de cette espèce dans un environnement pollué n'a donc pas de rapport avec la pollution elle-même.

Textularia truncata indique une influence marine forte alors que $P$. mediterranensis et $L$. lobatula, qui sont des espèces épibiotes, indiquent la présence de supports (algues ou mollusques), nécessitant eux-mêmes une influence marine forte. $P$. mediterranensis et $L$. lobatula sont corrélées négativement avec tous les contaminants, les corrélations étant les plus fortes avec $\mathrm{Ni}, \mathrm{Cd}$ et $\mathrm{Cr}$. 


\section{Conclusion}

Grâce à la connaissance de la répartition des foraminifères dans les systèmes paraliques, il est possible de les utiliser pour mettre en évidence des perturbations de l'écosystème, et en particulier celles dues aux impacts anthropogéniques. L'étude des foraminifères à différentes échelles d' organisation (peuplement, espèce ou individu) permet de recueillir suffisamment d'informations pour caractériser l'environnement et localiser les zones perturbées. La connaissance du comportement de ces organismes dans les estuaires et dans les ports permet d'envisager leur utilisation en routine comme indicateurs de la qualité du milieu dans un avenir très proche.

\section{Remerciements}

Ce travail a été possible grâce au support du Conseil Général de Vendée. Les analyses chimiques concernant le port de Port-Joinvilie ont été obtenues par la convention "Étude des sédiments portuaires, Conseil Général de la Vendée/IFREMER, port de Port-Joinville, 1998”. Les auteurs remercient R. Soulard et H. Grossel pour avoir fourni les échantillions et les analyses de PortJoinville. İls remercient également la cellule "Qualité des Eaux Littorales" de St Jean de Luz, et particulièrement M. Soulier et M. Raphos pour l'aide apportée au cours de l'étude de l'estuaire de l'Adour.

\section{Références bibliographiques}

ALVE E. (1991) - Benthic foraminifera in sediment cores reflecting heavy metal pollution in Sorfjord, Western Norway. Journal of Foraminiferal Research, 21, pp. 1-19.

ALVE E. (1995) - Benthic foraminifera response to estuarine pollution: a review, Journal of Foraminiferal Research, 25, pp.190-203.

BANDY O.L., INGLE J.C. et RESIG J.M. (1964) - Foraminifera: Los Angeles County outfall area, California, Limnology and Oceanography, 9, pp. 124-137.

BERNHARD J.M., (1986) - Characteristic assemblages and morphologies of benthic foraminifera from anoxic, organic-rich deposits: Jurassic through Holocene, Journal of Foraminiferal Research 16, pp. 207-215.

CASAMAJOR M.N. de et DEBENAY J.-P. (1995) - Les Foraminifères, bioindicateurs des environnements paraliques: reaction à divers types de pollution dans l'estuaire de l'Adour, ANPP-Colloque International Marqueurs Biologiques de Pollution, Abstracts Volume, Chinon-France, pp.371-377.

CATO I., OLSSONL. et ROSENBERG R. (1980) Recovery and decontamintation of estuaries. In Chemistry and Biogeochemistry of Estuaries, (eds. E. Ollauson, \& I. Cato), John Wiley and Sons Ltd., Chichester, pp. 403-440.

CLAISSE D. (1991) - Contamination chimique de l'Adour de la Nivelle et de la Bidassoa : bilan des observations du RNO (matière vivante), IFREMER, Nantes, $38 \mathrm{p}$.

DEBENAY J.-P. (1990) - Recent foraminiferal assemblages and their distribution related to environmental stress in the paralic environments of West Africa (Cape Timiris to Ebrie Lagoon), J. foram. Res. 20, pp. 267-282.

DEBENAY J.-P., ANDRE O., BEZIE S. et RAMBAUDS. (1997) - Foraminifers used as bioindicators in La Turballe Harbour (Loire Atlantique, France), Coastal 
zone monitoring and medium to long term forecasting, French-Japanise International Symposium, Paris, Abstracts Volume.

ELBEE (d') J. (1982) - Introduction à l'étude du zooplancton dans l'estuaire de l'Adour, Rapport de DEA, Institut Universitaire de Biologie Marine d'Arcachon, $38 \mathrm{p}$.

GESLIN E, (1999) - Impact des stress environnementaux sur les peuplements, la morphologie et la texture des foraminifères paraliques: implications pour leur utilisation comme bio-indicateurs. Thèse doctorat, Université d'Angers, 269 p.

HAYWARD B.W. et HOLLIS C.J. (1994) - Brackish foraminifera in NewZealand: A taxonomic and ecologic review, Micropaleontology 40, pp. 185-222.

LE FURGEY A. et ST JEAN J. Jr. (1976) - Foraminifera in brachish-water ponds designed for waste control and aquaculture studies in North Carolina, J. Foram. Res., 6(4), pp. 274-294.

LEGRAND H. et ROBBED. (1994) - Ports de plaisance, vie à bord et qualité des eaux, Colloque "de l'étude à l'aménagement des eaux lagunaires et côtières" Sète, Résumé.

MULLER W.A. et LEE J.J. (1969) - Apparent Indispensability of Bacteria in Foraminiferan Nutrition. J. Protozool., 16, pp. 471-478.

MULLINS H.T., THOMPSON J.B., McDOUGALLK. et VERCOUTERE T.L. (1985) - Oxygen minimum zone edge effects: evidence from the central California coastal upwelling system, Geology, 13, pp. 491-494.

MURRAY J.W. (1968) - Living foraminifers of lagoons and estuaries, Micropaleontology 14, pp. 435-455.

MURRAY J.W. (1991) - Ecology and paleoecology of benthic foraminifera, Longman, Harlow, 397 p.

NAIDU T.Y., RAO D.C. et RAO M.S. (1985) - Foraminifera as pollution indicators in the Vissakhapatnam Harbour Complex, east coast of India, Bulletin of Geological, Mining and Metallurgical Society of India, 52, pp. 88-96.

REDOIS F. et DEBENAY J.-P. (1996) - Influence du confinement sur la répartition des foraminifères benthiques. Exemple de l'estran d'une ria mésotidale de Bretagne méridionale. Revue de Paléobiologie, Genève 15, pp. 243-260.

ROUVILLOIS A. (1972) - Biocoenose des foraminifères en relation avec les conditions physico-chimiques du milieu dans les bassins et l'avant-port de SaintMalo (Ile et Vilaine), Cahiers de micropaléontologie ; ser. 3, 1, pp. 1-10, 2pls.

SHARIFI A.R., CROUDACE I.W. et AUSTIN R.L. (1991) - Benthic foraminiferids as pollution indicators in Southampton Water, Southern England, Journal of Micropaleontology, 10 (1), pp. 109-113.

SCHAFER C.T. (1970) - Studies of benthic foraminifera in Restigouche Estuary: Faunal distribution patterns near pollution sources, Maritime Sediments, 6, pp. 121-134.

SCHAFER C.T., COLLINS E.S. et SMITH J.N. (1991) - Relationship of foraminifera and thecamoebian distributions to sediments contaminated by pulp mill effluent: Saguenay Fiord, Quebec, Canada, Marine Micropaleontology, 17, pp. 255-283.

SCOTT D.B. et MEDIOLIF.S. (1986) - Foraminifera as sea level indicators. In van de Plassche (ed.), Sea level research: a manual for the collection and evaluation of data. Geo Books, Norwich, pp. 435-455.

STOUFF V., GESLIN E., DEBENAY J-P., LESOURD M. (1999) - Origin of morphological abnormalities in Ammonia (Foraminifera): studies in laboratory and natural environments. Journal of Foraminiferal Research, 29 (2) : 152170.

YANKO V., ARNOLD A. et PARKER W. (1999) - Effect of marine pollution on benthic foraminifera, In Modern Foraminifera (Sen Gupta ed.) Kluwer acad. Pub. $384 \mathrm{p}$. 